\title{
Less Invasive Management of A Wedged Impacted Tooth
}

\author{
Dr Mathew Tharakan ${ }^{1}$ MDS (OMFS), MDS (Orthodontics), MOSRCSED, \\ Dr Surej Kumar LK ${ }^{2}$ MDS \\ ${ }^{1}$ AssociateProf; Department of Oral and Maxillofacial Surgery PMS Dental College Thiruvananthapuram \\ INDIA \\ ${ }^{2}$ Prof \&Head; Department of Oral and Maxillofacial SurgeryPMS Dental CollegeThiruvananthapuram INDIA
}

\begin{abstract}
Though most of the literature relating to impacted tooth mentions about third molars and canines, the lower second molars has about 24 percentage chance of getting impacted among the other teeth, there is scarcity of articles related to wedged impacted lower premolars and their management.Surgical removal of wedged impacted tooth carries double the risk of adjacent tooth damage which will inversely affect the long term prognosis of the neighbouring teeth and surrounding structures, especially if carried out at an early age as in orthodontic correction. This article describes a case where a wedged impacted tooth was corrected non surgically and discusses the merits and demerits
\end{abstract}

Keywords: impacted tooth, less invasive, management

\section{Introduction}

Though the history ofimpactedtooth dates back to centuries they came to limelight after the invention of radiographs in 1895 and the subsequent development in radiology. Since then various diagnostic and treatment options have been advocated for the impacted tooth. With the latest developments in medical field more precise localisation and better management of the impacted teeth are possible now a days. Lower secondpremolars carry around 24 percentage chance of getting impacted among the other teeth in the mouth excluding third molars ${ }^{[1-3]}$, Mandibular second premolars rank third after permanent 3rd molars and maxillary canines, in frequency of impacted teeth ${ }^{[4]}$. Overall prevalence of impacted premolars is $0.5 \%$ and that of mandibular premolars is 0.2 to $0.3 \%{ }^{[4-5]}$. Various etiologic factors have been proposed regarding the second premolar impaction which mainly includes arch length discrepancies of various reasons like premature loss of posterior deciduous teeth, mesial migration of permanent posteriorteeth, and delayed eruption of posterior teeth. ${ }^{[4-5]}$ Traditional treatment optionsmainly advocated surgical removal of the wedged premolars. There is scarcity of reviews regarding orthodontic correction of such tooth. Though there are published protocols for managing impacted canines ${ }^{[6-9]}$, rarely any proposals for managing wedged premolars were found in the literature. Most of the times during surgical removal of wedged impactedteeth there are possibilities for damaging the anterior / posterior or both the teeth. If the damage occurs at an early age it will jeopardise the long term prognosis of the neighbouring teeth and that of the completedtreatment. On the contrary if the impacted tooth is allowed to erupt out physiologically or orthodonticallybrought toocclusion after creating space by removing an already erupted tooth where the surgical procedure is less traumatic to the local area, the long term prognosis will be far better. Here we report a case of wedged premolar treated non-invasively by intervening at the right time

\section{Case Report}

A 13 year old female patient reportedto the maxillofacial clinic for surgical removal of impacted lower right second premolar from elsewhere for orthodontic reasons. On clinical and radiographic examination she was diagnosed having a Skeletal 1 malocclusion with crowded upper and lower Labial segments, upper right canine (13) buccally erupted with total lack of space, impacted lower right second premolar with close proximity to first molar and premolar and incomplete root formation of impacted 45. ( Fig 1-Pre-treatment cast , Fig 2- pre-treatment OPG ) After consultation with the orthodontist the treatment plan needed unilateral upper and lower premolar removal for the orthodontic correction, as the OPG showed close proximity of the impacted tooth with the neighbouring teeth and incomplete root formation of the impacted tooth., it was decided to remove the $1^{\text {st }}$ premolar from lower jaw and to allow the second premolar for physiological / forced eruption .Immediatelyfollowing the extraction ofthe Right side upper and lower premolars, orthodontic treatment was started. within 3 months of treatment we could see the impacted tooth physiologically emerging out sufficiently to be attached to the lower arch wire. This was then bought into occlusion by orthodontic traction. (Fig 3- post treatment cast, Fig 4- post treatment OPG) 


\section{Discussion}

Lower second premolars take around 24 percentchance of getting impacted in the oral cavity among other teeth excluding third molars ${ }^{[3,4]}$. The prominent reasons for the Second premolars for getting impacted are because of the premature loss of deciduous second molars. The mesial migration of the permanent first molars which happens as a sequel will lead to space deficiency and cause the second premolars to get blocked or impacted, if corrective measures are not undertaken at the proper time ${ }^{[6-7]}$. There is no favourability of gender associated with the aetiology orincidence of impacted premolars .Various diagnostic measures ranging from simple IOPA(Intra oral periapical ) radiograph , shift cone technique, OPG(Ortho pantamogram), 3D(three dimensional)OPG,CT(Computed Tomogram), CBCT(Cone Beam Computed Tomogram) to 3D CT can be utilised for the precise localisation of the impacted tooth. The treatment options includes surgical removal of the impacted tooth which is an invasive procedure, conservative managements like creating space and await physiologic eruption and forced eruption after surgical bonding .Pre-adolescent and adolescent period where the premolar roots are still in growing phase and if the tooth is obstructed by an already erupted tooth, it is preferable to remove the already erupted tooth which causes less trauma to the surrounding hard and soft tissues than surgically removing the impacted tooth which possess a higher chance of damage to the neighbouring teeth and related structures. Thisdamage can affect the long term prognosis of the neighbouring teeth as well as that of the undertaken treatment.The surgical trauma anticipated to the local surroundings for the wedged tooth removal mainly depends on the position, angulation and proximity of the wedged tooth to the neighbouring teeth and to the neurovascular bundle. Sometimes the impacted tooth might have already initiated some root resorption of the adjacenterupted tooth to which it is exerting pressure. If such a root resorption is evident preference should be given to remove the already damaged tooth than removing an intact impacted tooth and later do additional procedures on the damaged tooth. Due consideration should be given to the possibility of root exposure/ damage of the adjacent teeth while removing the wedged impacted tooth. If proximity to the neurovascular bundle is observed with the impacted tooth and if any surgical damage is anticipated, preference should be given to remove the erupted tooth and then to force erupt the impacted tooth. There are some circumstances like associated pathology with the impacted tooth, malformations of the impacted tooth etc. where removal of the impacted tooth has to be carried out even if the associated factors are not favourable.

The eruption of the wedged impacted tooth to the oral cavity is influenced by certain factors like the availability of space to erupt out, the angulation to the neighbouring tooth and most importantly the stage of root formation of the impacted tooth ${ }^{[4-7]}$ The initial space creation for the impacted wedged tooth could be carried out by simple space regaining appliances if the deficiency is mild. Depending upon the nature of the space deficiency either space regainers, extraction or both might be needed to open up the space for the impacted tooth to erupt out ${ }^{[5-7]}$. Most of the times if adequate space is created and the rootformation of the impacted tooth is not completed the tooth will physiologically erupt out to the oral cavity ${ }^{[10]}$. If the position of the impacted tooth is not favourable and the root formation is incomplete some measures should be taken to maintain the space and periodic radiographic examination is advisable to evaluate the status of the impacted tooth, if favourable can wait for physiologic eruption or else orthodontic forced eruption should be considered. If the root formation is completed and the position is favourable, periodic evaluation can be considered initially after maintaining the space, and later if needed orthodontic eruption. If root formation is complete and the position is not favourable it is advisable to start with orthodontic eruption.

Advantages of physiological / forced eruption of impacted wedged tooth after removing the erupted tooth include ;Less invasive, minimal / nil damage to the neighbouring teeth and other structures, better long term prognosis of the neighbouring teeth and the undertaken treatment. Whereas the disadvantages to be mentioned are; Needs associated orthodontic intervention, anticipated longer duration of the treatment, better results expected when the roots of the embedded / impacted teeth are still growing

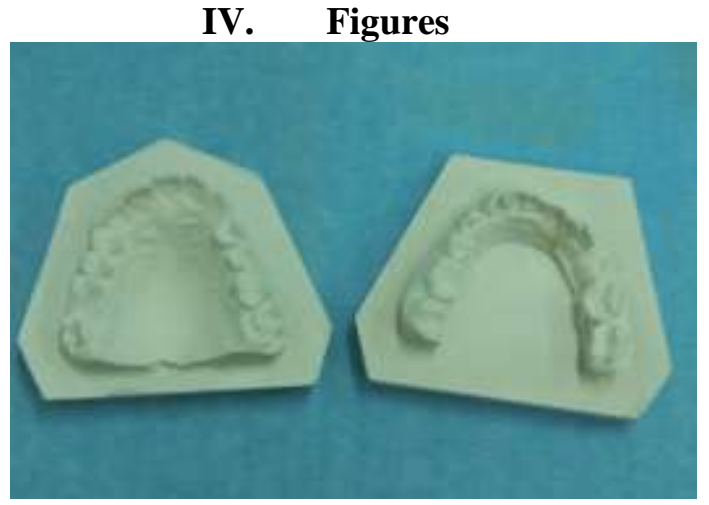

Figure 1. Pre-treatment cast 


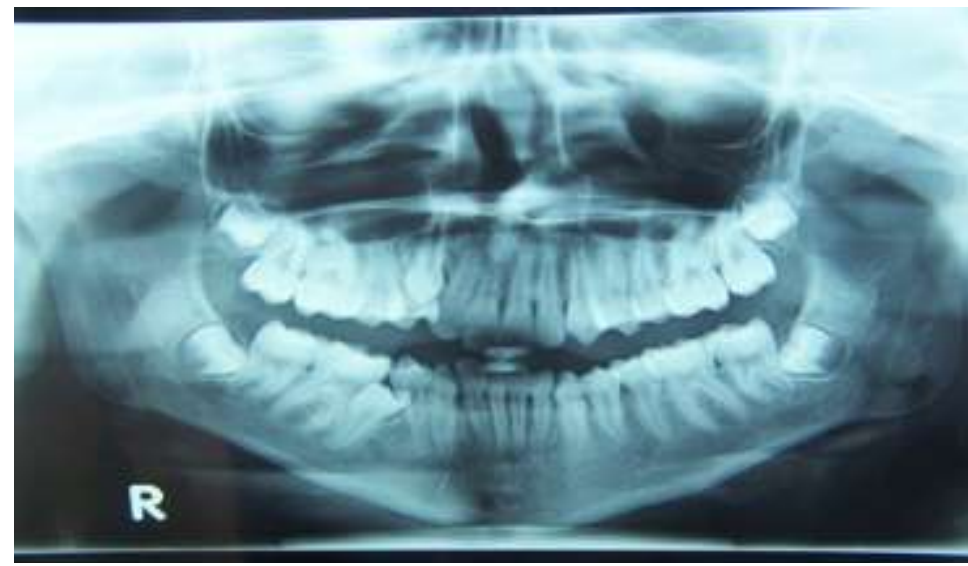

Figure2. Pre-treat OPG

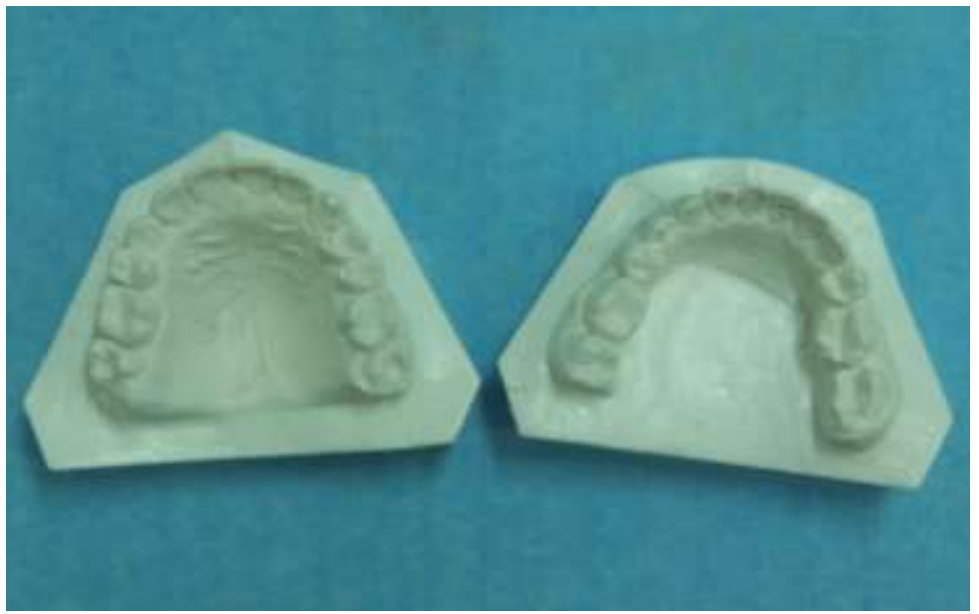

Figure3. Post treatment OPG

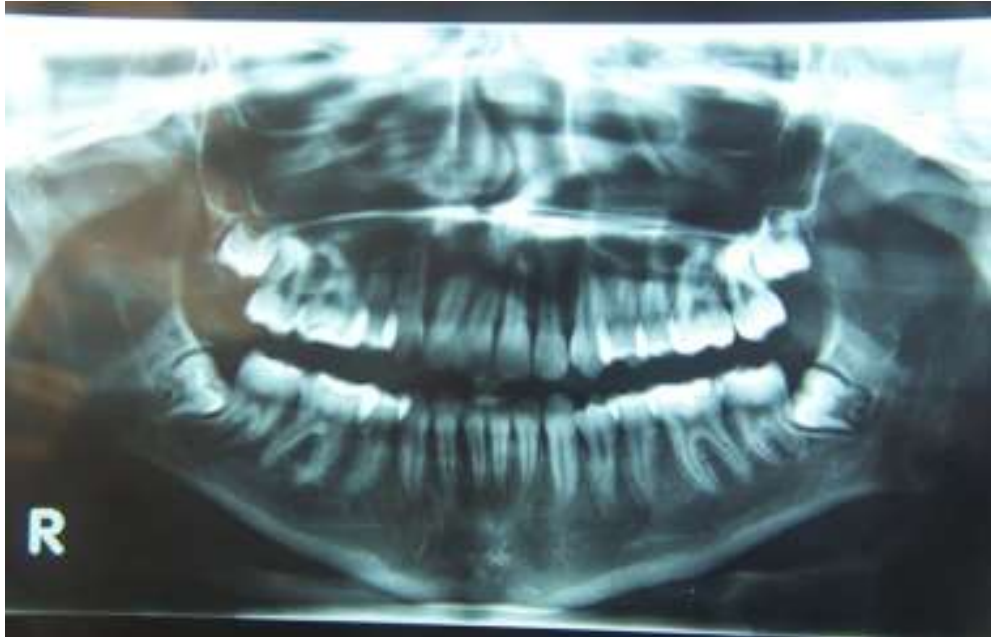

Figure 4. Post treatment OPG

\section{Conclusion}

Wedged impacted tooth especially second premolars is not uncommon to come across in the routine practice. Precise localisation, the relationship of such tooth to the neighbouring structures and non-invasive treatment options are possible nowadays due to the advances in technology.Treatment planning of such tooth should be done meticulously giving due consideration for the neighbouring teeth and surrounding structural integrity to avoid a surgical intervention, for a better long term prognosis, especially when observed and managed in the early adolescent ages . 


\section{References}

[1]. Anthony R Collett- conservative management of lower second premolar impaction. Australian dental journal 2000 ; 45 ;( 4); 279 281

[2]. AndreasenJO.The impacted premolar.In:AndreasenJO,PetersenJK,LaskinDM,eds.Textbook and color atlas of tooth impactions. Diagnosis, treatment and prevention. (Copenhagen: Munksgaard, 1997) 177-195.

[3]. Frank CA ; Treatment options for impacted teeth. J Am Dent Associ 2000; 131- 628-95

[4]. Jain U, KalluryA. Conservative Management of Mandibular Second Premolar Impaction. J Scient Res 2011; 4: 59-61.

[5]. Kalia V AneyaM ; Mandibular premolar Impaction ;Schol Res Erch 2009: 1-3

[6]. Willam R ProffitContemporary Orthodontics - 5 th Edition Complex non skeletal problems in preadolescent children preventive and interceptive treatment ; 470-490(Elsevier St. Louis, Missouri 2013)

[7]. Hupp JR, Ellis III E, Tucker MR. Contemporary Oral and Maxillofacial Surgery.Principlesof management of impacted teeth. 5th ed(Philadelphia: Elsevier publishers; 2008) 153-78.

[8]. Howard RD.Impacted tooth position: Unexpectedimprovements.Brit J Orthod 1978;5:87-92.

[9]. Yavuz MS, Aras MH, Buyukkurt MC, Tozoglu S. Impacted Mandibular Canines. J Contemp Dent Prac 2007; 8: 78-85.

[10]. McNamara C, McNamara TG. Mandibular premolar impaction: 2 case reports. J Can Dent Assoc. 2005;71(11):859-63 